\title{
APPLYING DATA MINING AND DATA VISUALIZATION WITHIN THE SCOPE OF AUDIO DATA USING SPOTIFY
}

\author{
Marika Apostolova Trpkovska, Arbesa Kajtazi, Lejla Abazi Bexheti and Arbana Kadriu \\ South East European University \\ Ilindenska n.335, 1200, Tetovo, Macedonia
}

\begin{abstract}
The aim of this research is to put forward an overview of applying data mining and data visualization within the scope of audio data from a dataset of Spotify. The research starts by presenting background information of these two fields and their influence on music industry. The paper includes explanation of the most essential concepts and their role. The research is concentrated on analysis of audio features of the tracks of Spotify's Top Songs in 2017 playlist and tries to highlight the common patterns behind the audio features of these songs. For the purposes of this research, Spotify datasets are used as practical scenario. For this reason, more detailed information is given about songs features, what are they, what do these top songs have in common and why do people like them. The result of the study showcase how singers and song makers can leverage the power of data visualization and data mining to help trying to predict one audio feature based on the others, look for patterns in the audio features of the songs and see which features correlate the most.
\end{abstract}

\section{KEYWORDS}

Data Mining, Data Visualization, Audio Data, Patterns, Spotify

\section{INTRODUCTION}

In the recent years, we started witnessing Big Data. This trend has influenced all industries. More and more data is generated and stored in different formats. There are many challenges we face with these exaggerated volumes of data, starting from the step of capturing them till properly displaying valuable information. "Data is the new oil. It's valuable, but if unrefined it cannot really be used. It has to be changed into gas, plastic, chemicals etc. to create a valuable entity that drives profitable activity; so data must be broken down, analyzed for it to have value". Big data comes with big promises. However consisting of only $1 \mathrm{~s}$ and $0 \mathrm{~s}$ the data is imperceptible to the public. For organizations to excel in their work, sometimes conducting only an analysis of data is not enough, therefore effective dissemination of the information is required.

The merit of data mining and visualization here is that they, together, utilize and make sense of the data. Since both fields are emerging, research on how to best combine these two areas is conducted every day. The field of music industry can benefit a lot from this synthesis. As new technology rapidly is being developed, the way of storytelling is changed by integrating data visualization tools.

The purpose of this research is to see what these songs have in common and why people like them by using big data analytics. Hopefully, with the new knowledge, it can contribute to the prediction of audio features based on others. To achieve this purpose, it is used a Spotify dataset where the audio features where extracted using the Spotify Web API and the Spotify Python library. The credit goes to Spotify for calculating the audio feature values. In doing so, this research will be of interest for the field of music industry where the organizations are trying to adapt to changes in the industry and for the scholars wanting to expand their knowledge in the application forms of data mining and data visualization.

\section{BACKGROUND}

Data mining is new powerful technology enhances the value of existing data by automating the process of extracting knowledge from it. By Rieger, massive databases are analyzed in minutes which enable users to 
conduct more studies in shorter amount of time and at the same time helps understand complex data. Data mining often is used as synonym for knowledge discovery from data (KDD), but in fact, it only depicts a step of that process.

Hidden patterns are found following various techniques and implementing the corresponding algorithms. Most popular techniques that provide diverse insights are: classification analysis, association rule learning, anomaly detection, clustering analysis and regression analysis. Data mining offers a solution to the problem by intelligently analyzing the present data in the database. This process often includes discovering patterns among data, finding specific connection between them that will be used for prediction of further actions.

The importance of data mining as resource is that it elucidates the hidden knowledge in large datasets. Applying data mining has proved to lead to new insights, better decision making and sometimes bring competitive advantage to companies. Data mining is interdisciplinary computer science field with broad implementation in other fields as well.

In scope of this, also the application of visualization provides unique perspective on the information and is often associated with coordinated system, points, lines, tables, charts or graphs. Additionally, it can be viewed as a form of humanizing statistics. The capability of combining data visualizations with stories gave birth to new form of storytelling known as "narrative visualization". Data visualization plays perhaps one of the most important roles in successfully communicating information. From human-computer interaction perspective, it has an ability to capture the attention of the human eye and brain. Ware explains the crucial role that it has by proclaiming that "Visual displays provide the highest bandwidth channel from the computer to the human. We acquire more information through vision than through all of the other senses combined. The 20 billion or so neurons of the brain devoted to analyzing visual information provide a pattern-finding mechanism that is a fundamental component in much of our cognitive activity.".

The two main reasons to use visualization as a form of presentation in stories by Goldman are: (1) exploratory: to understand what is present in the data; (2) communicative: to display and discuss visualizations with the public by telling stories based on the data and the facts.

Surprisingly, the question of "Why we like or not a particular song?" has received little attention from music psychology. Although music preference is recognized as a central aspect of modern identities, the field is "still in its infancy". The issue of liking per se is indeed difficult to study directly, and music psychologists have traditionally focused on less elusive, more directly measurable phenomena such as memorization, recognition or learning.

In our context, a central issue in trying to explain music hits is exposure, that is, the simple fact of listening to a musical piece. What is the effect of exposure on preference or liking? Studies on exposure show that there is indeed an impact of repeated exposure on liking, but also that this impact is far from simple. Parameters such as the context, type of music or listening conditions (focused or incidental), seem to influence the nature of this impact, and many contradictory results have been published. The popular idea that repeated exposure tends to increase liking was put forward early and was confirmed experimentally in a wide variety of contexts and musical genres. The so-called mere exposure effect, akin to the familiarity principle, or perceptual fluency, is considered by many psychologists to be a robust principle, pervading many facets of music listening.

However, as noted by Schellenberg, this increase in liking may be restricted to musically impoverished or highly controlled stimuli. Indeed, other studies have shown a more subtle effect of repeated exposure. The study by Siu-Lan et al. showed different effects of exposure on intact and patchwork compositions. An inverted U-curve phenomena was observed in particular by Szpunar et al. and Schellenberg in his journal article, it explained in large part by the "two factor model". In this model, two forces compete to build up liking: (1) the arousal potential of the stimulus (the music), which decreases with repeated listening, thereby increasing liking (with the habituation to this arousal potential), and (2) familiarity, which tends to create boredom. These two forces combined produce typical inverted U-shapes that have been observed in many studies of preference. This model is itself related to the famous "Wundt curve". The Wundt curve describes the typical experience of arousal as being optimal when achieving a compromise between repetition/boredom and surprise.

Yet, other studies performed by Witvliet showed in contrast a polarization effect, whereby repeated exposure does not influence initial likings but makes them stronger, both positively and negatively. At last, Loui et al. studied exposure effects by considering exotic musical temperaments, to study the relation between learning and preference. They showed that passive exposure to melodies built in an entirely new musical system led to learning and generalization, as well as increased preference for repeated melodies. 
This work emphasizes the importance of learning in music preference. These psychological experiments show that a relation between exposure and liking exists, but that this relation is complex and still not well understood, in particular for rich, emotionally meaningful pieces. It is therefore impossible to simply consider, from a psychological point of view, that repeated exposure necessarily increases liking: it all depends on a variety of factors.

Big data is a term that reflects the amount of information people generate. Some estimate that today, humans generates more information in one minute than in every moment from the earliest historical record through 2000. Unsurprisingly, harnessing this data has shaped music industry in radical new ways. With data becoming easier to acquire and analyze, it has now become possible to crunch large numbers and predict audience preference and buying tendencies. Whereas in the past, the industry relied primarily on sales and how often a song was played on the radio, they can now see what specific songs people are listening to, where are they hearing it and how they are consuming it. Big Data is being integrated into nearly every field. It should be no surprise that the multi-billion dollar music industry wants in. There are two major ways big data is already influencing the music industry; music creation and music selection.

There is a certain circular quality to pop music. The more you are forced to listen to that inescapable new song, the more you like it. You liking means more music of the same type will be produced. Data, however, is making the new era of music one of the most populist. For example; in our case Spotify knows what listeners like and want. The ability to listen to music is only the most basic feature of Spotify. They constant compile data and create algorithms to suggest new music to listeners.

\section{PROBLEM STATEMENT}

The music industry is in the middle of something new and bold, thanks to digital technology. Traditional distribution venues have already been replaced with digital ones and this has surprisingly boosted the popularity of artists. Instead of slowing things down, downloads and media streaming have helped artist mount more successful tours and sold out concerts than ever before. This is a positive example of what digital (data-driven) innovations and bold ideas can do.

From a global music currency to virtual multi-camera recording setups, there are a lot of ways that technology is helping the music industry grow. Fifteen years ago, Steve Jobs introduced the iPod. Since then, most music fans have understood this has radically changed how they listen to music. Data from what we download and listen to can now be mined to create and promote future songs. Less understood are the ways that raw information - accumulated via downloads apps and online searches - is influencing not only what songs are marketed and sold, but which songs become hits.

Decisions about how to market and sell music, to some extent, still hinge upon subjective assumptions about what sounds good to an executive, or which artists might be easier to market.

Increasingly, however, businesses are turning to big data and the analytics that can help turn this information into actions. But, the big question raised in this research is: Can someone predict whether your recently produced song will become a hit?. Any pop song composer would probably laugh at this question and respond: How could someone predict the success of what took so much craft, pain, and immeasurable creativity to produce?. This question raises a recurring fantasy in every culture: wouldn't it be thrilling to understand the "laws of attraction" that explain how this sort of preference system of music in human beings works, to the point of being able to predict the success of a song or any other cultural artifact before it is even released? This fantasy is elaborated in detail in Malcom Gladwell's story "The Formula". In this fiction, a system is able to predict the success of movies by analyzing their script in automatic way. The system is even smart enough to propose modifications of the script to increase the success of the movie, with a quantified estimation of the impact in revenues.

This process is also true for music: what makes us like a song or not probably has to do with a complex combination of micro-emotions, themselves related to our personal history, to the specifics of the song and too many other elusive elements that escape our direct understanding.

However daring, we challenged this assumption by precisely undertaking the task of making these kinds of predictions. Researches with similar background of a predictive analysis with data visualization could not be found just some of them were only researches without including data mining and data visualization. There is one project on Kaggle in python that is really close to this research but, that used different kind of techniques for an attempt to build a classifier that can predict whether or not he likes a song. 


\section{METHOD}

For this research, a traditional knowledge discovery process was used: first the data was gathered, pre-processed and cleaned, analyzed and at the end the results were visualized. The general system architecture followed is presented in Figure 1.

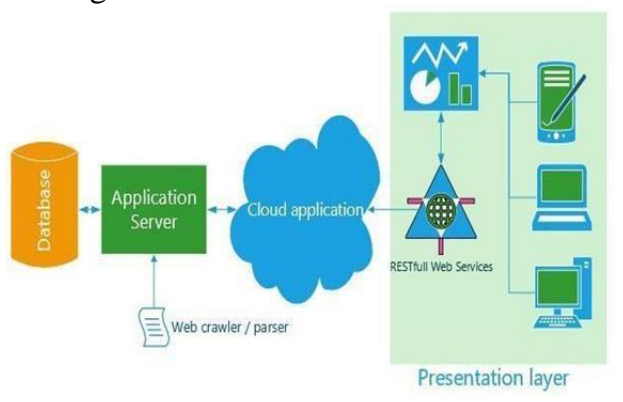

Figure 1. General system architecture

Even though the data is open and free, there are costs associated with the process of getting the dataset as a resource. Often the data should be converted into specific format, annotated or by necessity a certain metadata to be created. All of this requires human resources with specialized skills to do the work and technology resources (both hardware and software).

Spotify has developed a workflow manager, Luigi, which it is open source. Luigi is a Python framework for data flow definition and execution. Luigi is used to crunch a lot of data. Most of the data is user-centric data, such as billions of log messages that allows Spotify to provide music recommendations or select for example the next song heard on the radio. The data however is also used in decision-making, providing forecasting information and business analytics. So, the dataset was taken from Kaggle. The dataset contains the daily ranking of the 200 most listened songs in 53 songs from 2017 and 2018 by most Spotify users. It contains more than 2 million rows, which comprises 6629 artists, 18598 songs for a total count of one hundred five billion streams count. The data spans from 1st January 2017 to 9th January 2018 and will be kept up-to-date on following versions. It has been collected from Spotify's regional chart data.

For manipulating with data and visualizing the results, RStudio as integrated development environment (IDE) for $\mathrm{R}$ was used. $\mathrm{R}$ is designed to read entire datasets into memory and work with them there. The dataset imported in RStudio was of .csv format. For some of the cases, it was necessary to read the data into a data frames and use that data frame to conduct further manipulations. Data visualization was possible with the use of ggplot2 and plotrix package. This plotting system of $\mathrm{R}$ allows customization of the diagram and offers different built-in functions. Combination of data, aesthetic mappings and geom (geometric object) are specified for every diagram. The details of how data values are translated into visual representation were controlled by changing the scales, limiting the values of the axes, modifying labels and legend and adding theme to the plot.

\subsection{Results}

First of all the duration is converted in seconds for the need to simplify the analysis (Figure 2). Here we create a variable where we get the column "duration_ms" and convert it into seconds with a name of "duration_s".

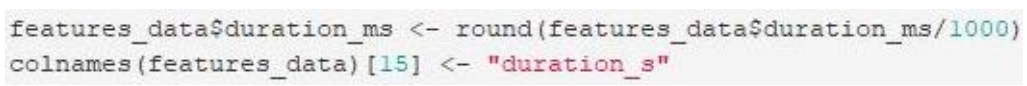

Figure 2. Converting the Duration in Seconds

On the next step the bar graph on Figure 3 shows "top artists based on their appearance and songs" and the $\mathrm{R}$ code. 


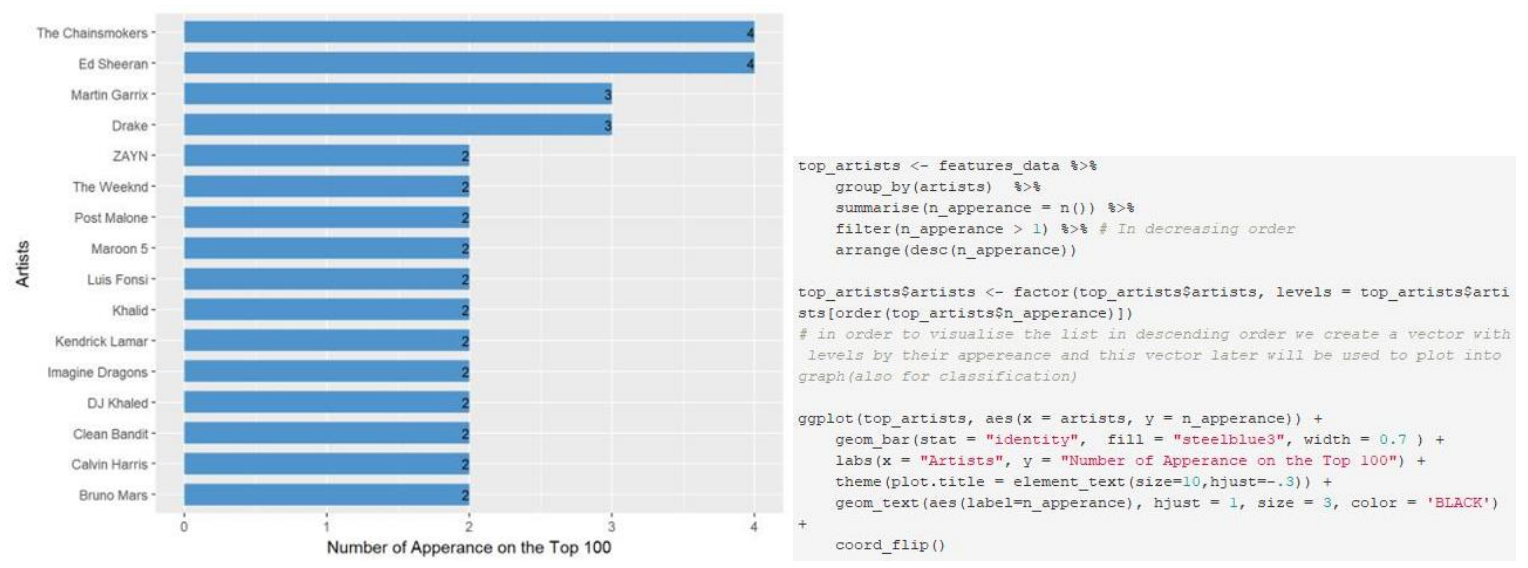

Figure 3. Top Artist Based on their Appearance and the R Code

Here firstly we create a variable top artists and took the dataset features_data to group all the artists where and summarize all the appearance in the data set of the artist and filter them in descending order.

For creating the graph we used ggplot where we use the list top artists we created and with two axes, $\mathrm{x}$ which equals to artists and $\mathrm{y}$ which equals the appearance of an artist. Then we use the $\mathrm{R}$ studio features to design the graph with the colors and other features we like.

The graph on Figure 4 below shows the Top Artist in Central Europe by playing time.
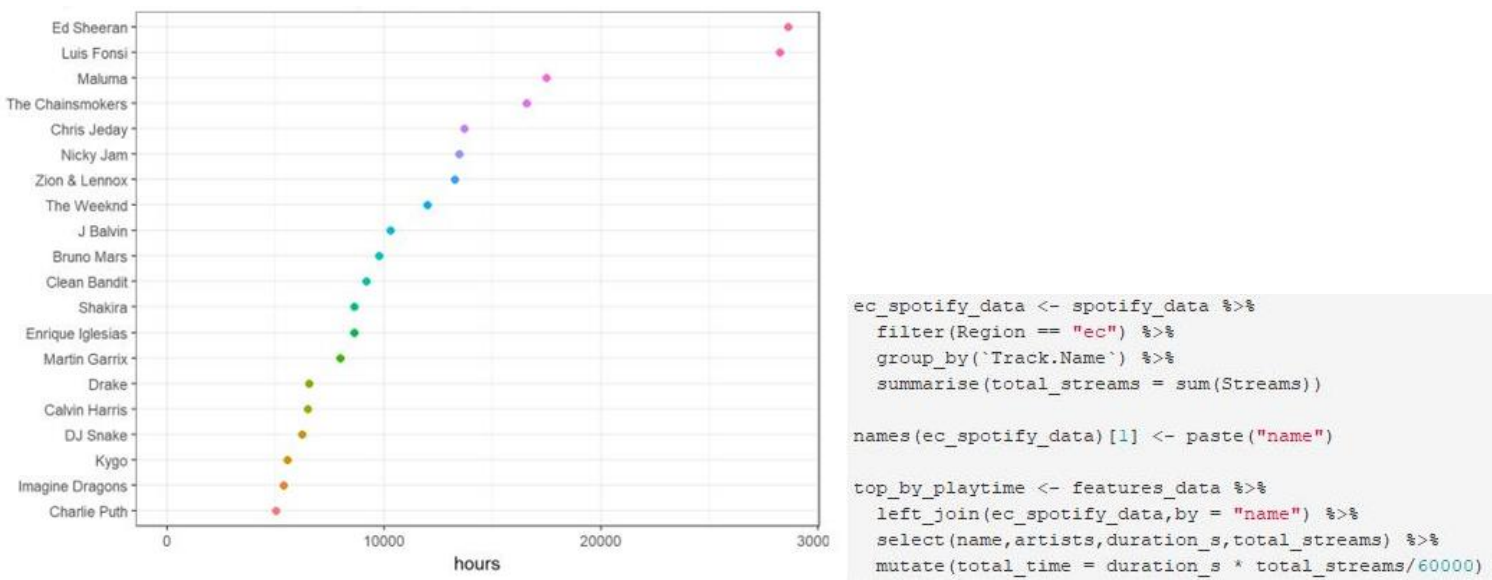

Figure 4. Top Artists in Central Europe by Paying Time and the R Code

As we can see on the above graph the artist Ed Sheeran was played the most by the users, reaching nearly 30000 hours played, followed by artist Luis Fonsi and so on going down. From the graph we can see that artists like Lois Fonsi and Maluma and Chris Jeday now started to be on top of the list.

For Top Artist by playing time in this case we used the "Streams" variable of Spotify_data and "duration" of features_data, data sets.

Here at the first code we create a new list with the name top20_by_playtime, by using the list top_by_playtime we group by the artist's name, create a variable n_time where we save the sum from the total_time and arrange by descending order.

In the below graph (Figure 5) we visualized finding the relationship between the variables of the features_data dataset. 


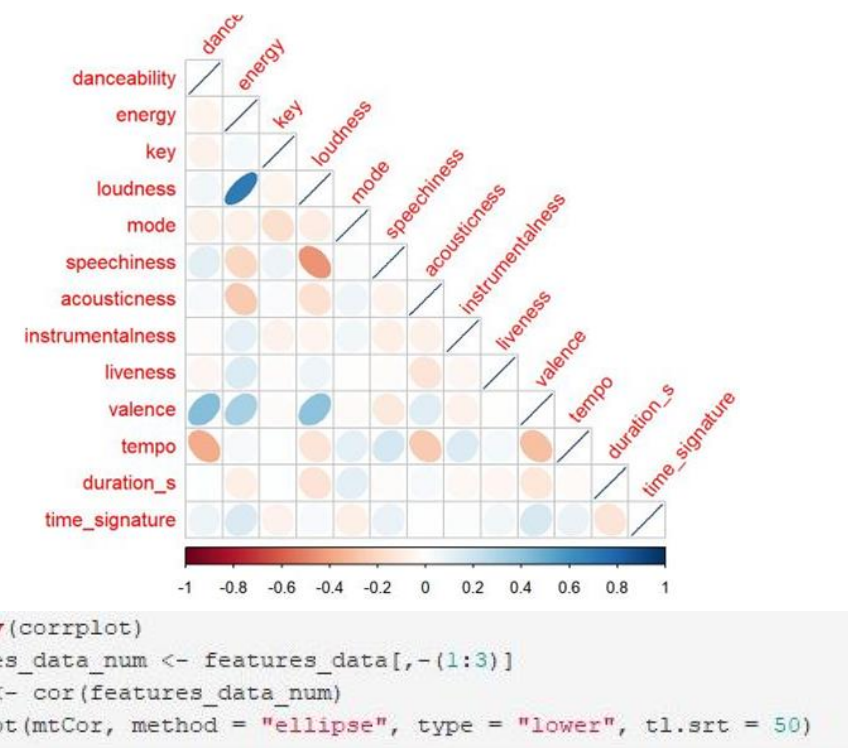

Figure 5. The Relationship between the Variables and the R Code

After finding the relationship between the variables we can conclude that "energy" and "loudness" are highly positively correlated. Also "valence" is more correlated with "danceability, energy and loudness". The variable "loudness" and "speechness", "tempo" and "danceability", "tempo" and "valence" are negatively correlated.

Here we used the library corrplot for visualizing the relationship between the variables. Will create a new variable fetures_data_num and will avoid the three first columns because the first columns are not numeric and include the name of the artist, song etc. And then we will use the method "ellipse" of type "lower". On Figure 6 is shown the distribution of the above 3 variables and how are they distributed among top 100 songs.

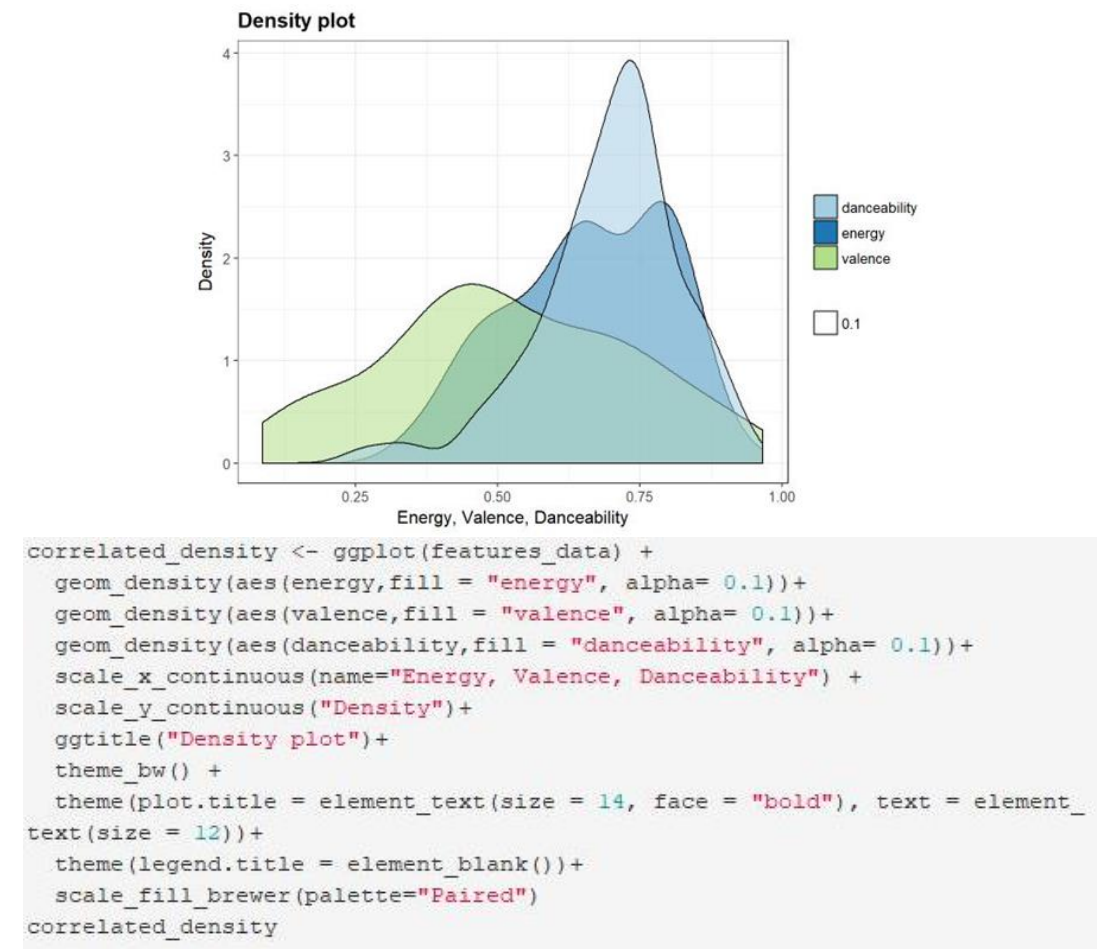

Figure 6. The distribution of Energy, Valence and Danceability and the R code 
We created a variable correlated_density where this variable contains the ggplot of features_data datasets then we find the density of the variables energy, valence and danceability and we use the R studio features to design the graph depending on our preferences.

Among all the 13 Keys that were appeared on this top 100 songs in Spotify by users we counted the amount of time each key song appeared the most (Figure 7).
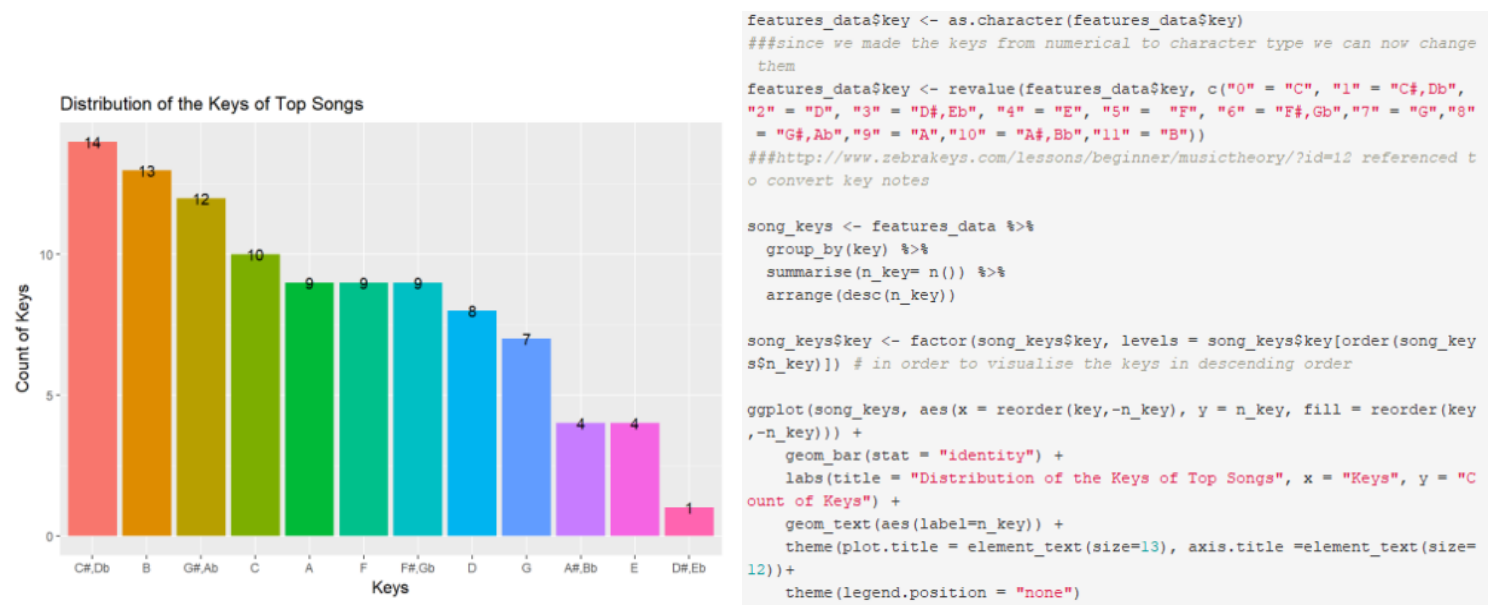

Figure 7. Distribution of the Keys of Top Songs and the R Code

For the benefit of analysis, we converted the keys into their original symbols. First, here we create a variable fetures_data\$key where we convert the keys into their original symbols. After that we create another variable where we summarize all the keys and arrange by descending order. We then use the ggplot to show the distribution of the keys and here from the graph we can see that the note C\# was the one that appeared the most on the top 100 list of Spotify songs played by users.

On the graph presented on Figure 8 we used a Decision tree analysis to see which of the songs have the highest chances to be on the top 100 list played by users.

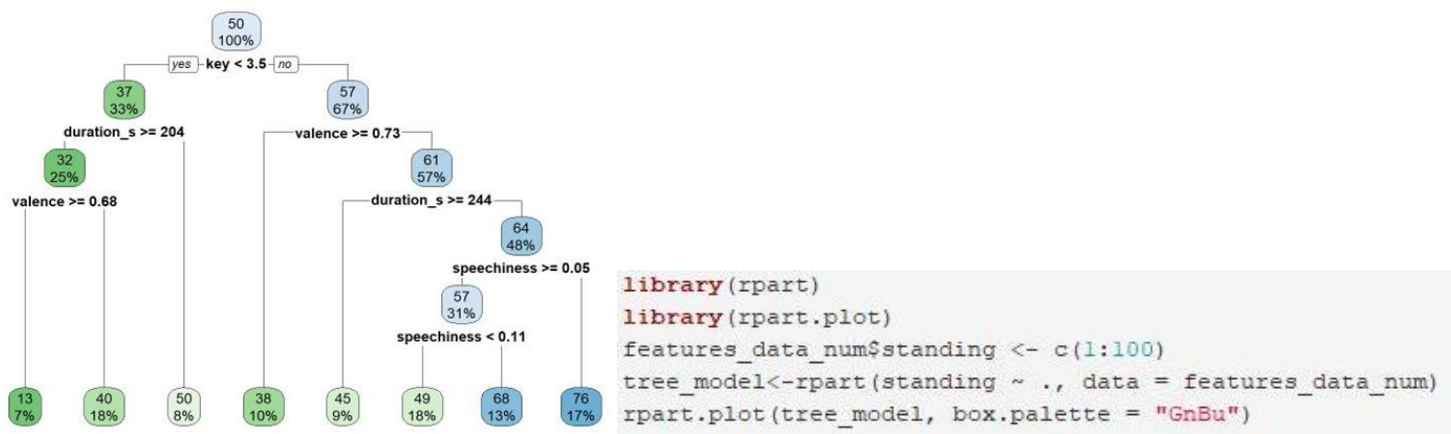

Figure 8. Decision Tree and the R Code

First, for conducting a decision tree analysis we need two libraries rpart and rpart.plot. Then, create a list features_data_num\$standing with all top 100 songs and then, create a variable tree model where we generate the decision tree model.

\section{CONCLUSION}

The main purpose of this research was to put forward an overview of applying data mining and data visualization within the scope of audio data from a dataset of Spotify. The paper presented explanation of the most essential concepts and their role in this field. By the analysis of audio features of the tracks of Spotify's Top Songs in 2017 playlist were highlighted the common patterns behind the audio features of these songs. 
A practical scenario was detailed explained. The results of the study showed how singers and song makers can leverage the power of data visualization and data mining, supporting the prediction of one audio feature based on the others, looking for patterns in the audio features of the songs and seeing which features correlate the most. We can conclude that the songs on which the key values are less than 3.5 ('C', 'C\#', 'D', 'D\#', 'Eb'), Duration is more than 204 seconds and Valence is more than 0.68 have highest chance to be around the Top 100 list.

\section{REFERENCES}

Rieger, A., 2011. Large scale data analysis and predictive modeling in data mining. Bosch ConnectedWorld Blog, [online] available at: https://blog.bosch-si.com/developer/large-scale-data-analysis-and-predictive-modeling-in-datamining/

Gladwell, M., 2006. The formula. The New Yorker, [online] available at: https://www.newyorker.com/ magazine/2006/10/16/the-formula

Goldman, W., 1983. Adventures in the Screen Trade. Warner Books, New York.

Loui, P., et al, 2010. Humans rapidly learn grammatical structure in a new musical scale. Music Perception, 25(5), pp. 377-388.

Spotify Charts, 2018. [online] available at: https://spotifycharts.com/regional

GeorgeMcIntire, 2017. Spotify Song Attributes | Kaggle. [online] available at: www.kaggle.com/geomack/ spotifyclassification/data

Schellenberg, E.G., 2008. The role of exposure in emotional responses to music. Behavioral and Brain Sciences.

Schellenberg, E.G. et al, 2008. Liking for happy- and sad-sounding music: Effects of exposure. Cognition and Emotion Journal, 22(2), pp. 218-237, 2008.

Siu-Lan, T. et al, 2006. The effects of repeated exposure on liking and judgments of musical unity of intact and patchwork compositions. Music Perception: An Interdisciplinary Journal, Vol. 23 No. 5, pp. 407-421.

Szpunar, K.K. et al, 2004. Liking and memory for musical stimuli as a function of exposure. Journal of Experimental Psychology: Learning, Memory, and Cognition, 30(2), pp. 370-381.

Ware, C., 2013. Information Visualization: Perception for Design. Third Edition, Elsevier.

Witvliet C.V.O., et al, 2007. Play it again Sam: Repeated exposure to emotionally evocative music polarizes liking and smiling responses, and influences other affective reports, facial EMG, and heart rate. Cognition and Emotion Journal, 21(1), pp. 3-25.

Wundt, W., 1897. Outlines of Psychology. Englemann, Leipzig, Taylor \& Francis Group, LLC 